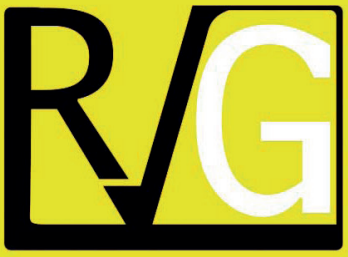

Julio - Septiembre, 2021

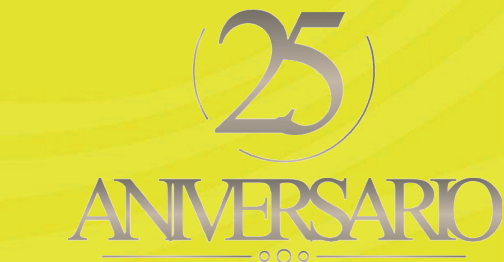

ANMERSARO
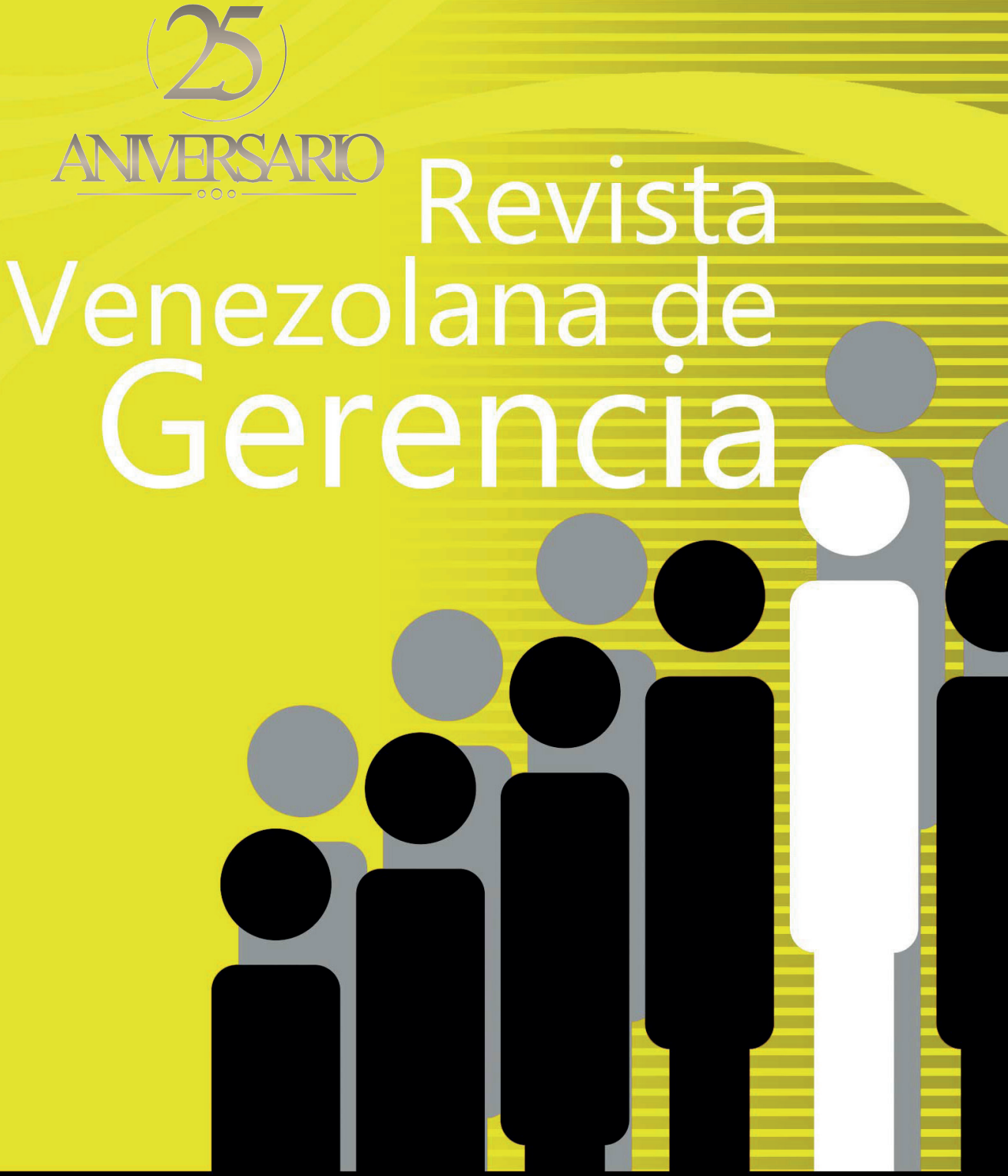

UNIVERSIDAD DEL ZULIA (LUZ)

Facultad de Ciencias Económicas y Sociales Centro de Estudios de la Empresa

ISSN 1315-99

Esta obra está bajo una licencia de Creative Comm Reconocimiento-NoComercial-Compartirlgual 3.0 Unpo http://creativecommons.org/licenses/by-nc-sa/3.0/deed.es 
COMO CITAR: Varguillas, C., Brizuela Camacho, S., Villarroel Dávila, P. C., y Gallardo V., D. A. (2021). Estrategias de aprendizaje e integración Curricular en la Universidad Técnica Particular de Loja en Ecuador. Revista Venezolana de Gerencia (RVG), 26(95), 818-831. https://doi.org/10.52080/eq.vol1. iss34.23
Universidad del Zulia (LUZ)

Revista Venezolana de Gerencia (RVG)

Año 26 No. 95 Julio-Septiembre 2021, 818-831

ISSN 1315-9984 / e-ISSN 2477-9423

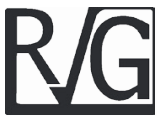

\title{
Estrategias de aprendizaje e integración Curricular en la Universidad Técnica Particular de Loja en Ecuador
}

\author{
Varguillas, Carmen* \\ Brizuela Camacho, Sor Natalia** \\ Villarroel Dávila, Paola Carina*** \\ Gallardo V., Daniel Armando****
}

\section{Resumen}

En la presente investigación se abordaron los mecanismos idóneos para la integración de los elementos que conforman la estructura curricular, basada en el enfoque por competencias. El objetivo fue describir el proceso de integración curricular mediante la creación de la cátedra integradora en la carrera de educación básica de la Universidad Técnica Particular de Loja en Ecuador, orientados a la definición de estrategias de aprendizaje para los actores involucrados. Se aplicó el método de investigación-acción-participativa; este permitió la indagación realizada por el profesorado, para promover el desarrollo de competencias en 85 estudiantes de la carrera de Educación Básica. Los resultados indican que en el diseño de estrategias de aprendizaje, se priorizó la creatividad de los involucrados para proponer estrategias pertinentes con la problemática detectada. Se concluye que los estudiantes valoran, la importancia de la integración de las asignaturas: Cátedra integradora 2 y Planteamiento de la investigación acción participativa porque, superan los esquemas tradicionales convirtiendo el proceso de aprendizaje en un sistema integral.

Palabras clave: integración curricular; cátedra integradora; educación básica; enfoque por competencias.

Recibido: 19-04-21

Aceptado: 15-06-21

* PhD. En Educación. Profesora e investigadora de la Universidad Nacional de Chimborazo UNACH- Facultad de Ciencias de la Educación, Humanas y Tecnologías. Ecuador. Email: cvarguillas@unach.edu.ec ORCID: https://orcid.org/0000-0002-3834-2474

** PhD En Educación. Docente Universidad de la Técnica Particular de Loja. Email: snbrizuela@utpl.edu.ec. ORCID: https://orcid.org/0000-0003-4347-0486

*** Magister en Educación Parvularia. Magister e Neuropsicología y educación. Docente de la Universidad Técnica Particular de Loja. Email: pcvillarroel@utpl.edu.ec. ORCID: https://orcid.org/0000-0003-4917-8304

**** Lic. En Ciencias Políticas y Administrativas. Cursante de la Maestría en Estudios Internacionales. Universidad Central de Venezuela. Email: danielarm.gallardo@gmail.com. ORCID: https://orcid.org/0000-0001-5250-3302 


\title{
Learning strategies and Curricular integration at the Private Technical University of Loja in Ecuador
}

\begin{abstract}
In this research, the ideal mechanisms for the integration of the elements that make up the curricular structure, based on the competency approach, were approached. The objective was to describe the process of curricular integration through the creation of the integrating chair in the basic education career of the Particular Technical University of Loja in Ecuador, oriented to the definition of learning strategies for the actors involved. The participatory action-research method was applied; This allowed the investigation carried out by the teachers, to promote the development of competencies in 85 students of the Basic Education career. The results indicate that in the design of learning strategies, the creativity of those involved was prioritized to propose pertinent strategies with the problem detected. It is concluded that the students value the importance of the integration of the subjects: Integrative Chair 2 and Approach to participatory action research because, they surpass the traditional schemes, turning the learning process into an integral system.
\end{abstract}

Keywords: curricular integration; integrative chair; basic education; competency approach.

\section{Introducción}

En las últimas décadas, la educación superior ha generado profundos debates sobre la necesidad de responder a las demandas de la sociedad y del campo laboral, con el fin de contribuir a la solución de problemáticas reales que mejoren las condiciones de vida humana y aporten a procesos de desarrollo sostenible en el entorno local y global.

El currículo tiene una dimensión de gestión muy importante para su desarrollo, por lo tanto, deben integrarse los criterios epistemológicos, psicológicos, pedagógicos, de evaluación, de organización académica, comunicativos y administrativos, para justificar lo que el currículo selecciona, crea, organiza, transmite y produce en el diseño y desarrollo de contenidos, metodologías y lenguajes para los programas (Casanova, Paredes y Ortega, 2020).

Los diseños curriculares en las instituciones de educación superior buscan generar escenarios contextualizados para vincular la teoría y la práctica e integrar de forma coherente los contenidos de las diferentes áreas del conocimiento científico y humanístico. Por ello, se considera pertinente un diseño curricular, donde se describan los, contenidos, competencias, recursos, 
Varguillas, Carmen; Brizuela Camacho, Sor Natalia; Villarroel Dávila, Paola Carina y

Gallardo V., Daniel Armando

Estrategias de aprendizaje e integración Curricular en la Universidad Técnica Particular de Loja en Ecuador

estrategias y evaluación, adaptado a la realidad.

En el contexto internacional la mayoría de propuestas de integración curricular se encuentran en la educación básica o bachillerato con el diseño de unidades didácticas integradas (Illán y Molina, 2011). Según Zamudio, Leiva y Fernández (2019), las propuestas de formación expresadas en los planes de estudio universitario han adoptado tradicionalmente una organización disciplinar fragmentada; como una meta por alcanzar y a la vez que representa un problema a resolver. Se considera necesario, indagar el estado de situación de las propuestas curriculares respecto de los niveles de integración, en las instituciones de educación universitaria e identificar un conjunto de variables relevantes asociadas a procesos y experiencias vigentes de integración curricular.

En Ecuador se planteó un modelo de rediseño curricular en el año 2014, bajo las directrices del Consejo de Educación Superior (CES), el mismo partió desde un enfoque sistémico, intercultural, multidimensional, en el cual, entre otras cosas, se pretendió una ruptura epistemológica, para el cambio de la formación exclusivamente disciplinar y fragmentada, a otras formas de organización del conocimiento de forma abierta e integrada.

$$
\text { Desde la racionalidad }
$$

interdisciplinar se sostiene la emergencia de un nuevo modelo que construya al desarrollo del sistema teórico-práctico, derribando fronteras disciplinares, donde se promuevan las relaciones, solidaridades y diálogo entre los saberes, la integración de estilos de pensamiento, la transversalidad en nuevos lenguajes, problemas y finalidades; transgrediendo métodos y barreras disciplinares
(Granados, 2014).

Estos aspectos se reflejan en la creación de una cátedra integradora en cada período académico que se encargue del estudio de las tensiones, problemas y situaciones específicas de la realidad que son objeto de la carrera. Esta cátedra debe encargarse de la planificación, análisis, evaluación y retroalimentación de las prácticas pre-profesionales. Los proyectos integradores de cada período académico, según Granados (2014:60) "deberán tener su espacio de gestión en esta cátedra e integrar plataformas o redes de aprendizaje con asignaturas del campo teórico, de investigación y de integración de saberes".

La concepción antes señalada, sin lugar a dudas, conlleva a la vinculación de teoría y práctica donde los estudiantes contextualicen su formación, implicándose con la realidad de manera participativa y proactiva, superando esquemas tradicionales y promoviendo aprendizajes significativos y perdurables. Por su parte, los docentes son actores de nuevas formas de construir conocimientos pedagógicos que innoven los procesos educativos y transformen las dinámicas sociales $y$ culturales. De acuerdo con Fernández (2004:8) "la construcción local del conocimiento se genera en el contexto de las respectivas comunidades. Los profesores pueden llegar a ser creadores e intérpretes activos del currículum, no simples implementadores".

Si bien estos planteamientos son muy prometedores, en la realidad, la mayoría de instituciones educativas deben recorrer un largo camino para llevar a cabo la integración curricular, como una forma de contextualizar el currículo prescrito con la intervención activa de los docentes en su diseño y organización. Los esfuerzos por el 
cumplimiento de esta aspiración son escasos, pues la defensa del espacio disciplinar se mantiene en las acciones de los actores educativos.

En la actualidad en algunos docentes, existe una concepción equivocada de trabajar aisladamente, porque resulta más fácil una organización lineal de contenidos que armar un sistema de habilidades cognitivas, procedimentales y actitudinales para aplicarse en función de un objetivo común. Se delegan responsabilidades individuales, pero no se asumen las colectivas, entonces se desvanece, la articulación y el accionar del proceso de enseñanza. Situación que se torna más evidente en la modalidad a distancia, en la cual el aprendizaje está mediado por aplicaciones tecnológicas y materiales didácticos digitalizados, donde existe limitado contacto presencial por lo que el educador no sólo debe ser capaz de aplicar las tecnologías al servicio de la educación, sino también, debe tener las habilidades y destrezas para diseñar nuevos escenarios educativos donde los estudiantes puedan aprender a interactuar en el espacio telemático (Sandoval, 2020).

Frente a estas circunstancias, la Universidad Técnica Particular de Loja (UTPL) en Ecuador, como institución con más de 50 años de trayectoria académica, ejerciendo procesos de formación, investigación, innovación, internacionalización y vinculación; apoyados en la implementación de nuevos enfoques, metodologías, estrategias y técnicas, para aplicar de forma innovadora lo que establece la malla curricular vigente. De allí que en la carrera de educación básica, durante el segundo período académico octubre 2019-febrero 2020, se asumió trabajar el aprendizaje basado en retos, como un camino para promover la integración curricular y la vinculación teóricapráctica. Este tipo de aprendizaje involucra al estudiante en un contexto real para resolver una problemática relacionada con el tema o temas de estudio (Malmqvist, Rådberg y Lundqvist, 2015).

El trabajo se enfocará en superar la visión disciplinar en la formación de los estudiantes de la carrera de educación básica, pues el enfoque academicista ha resaltado los conocimientos teóricos de cada asignatura sin integrarlos a la práctica, ni tampoco relacionarlos con las demás asignaturas, es decir no existe una relación dialógica interdisciplinar y transdisciplinar.

Por lo mencionado, el objetivo fue describir el proceso de integración curricular mediante la creación de la cátedra integradora en la carrera de educación básica de la Universidad Técnica Particular de Loja en Ecuador, proyectando estrategias orientadas al fortalecimiento de los procesos de aprendizaje para los estudiantes.

\section{Integración curricular}

La Integración Curricular, defiende una visión del aprendizaje y la enseñanza como un camino que profesores y alumnos deben seguir para construir conexiones significativas entre el mundo del aula y el mundo real más amplio (Molina, 2011). Desde esta perspectiva, la integración curricular responde a un modelo que progresivamente avanza desde la estructura fragmentada por disciplinas, hasta el nivel de máxima integración en modelos inter y transdisciplinares mediante tres dimensiones: la cognitiva, la epistemológica y la curricular (Zamudio, Leiva y Fernández, 2019) 
Para el desarrollo de la integración curricular se asume como estrategia el aprendizaje basado en retos, en el cual se involucra al estudiante en una situación problemática real, significativa relacionada con su entorno, lo que implica definir un reto e implementar una solución para éste (Observatorio de Innovación Educativa del Tecnológico de Monterrey, 2015). Se parte de un aprendizaje vivencial que conecta lo que los estudiantes aprenden en la institución de educación superior y lo que está en el medio social, fomentando además el desarrollo de habilidades en investigación.

El modelo pedagógico de la Universidad Técnica Particular de Loja está basado en competencias (UTPL), donde el estudiante es el actor principal del proceso educativo, en el caso de la modalidad a distancia el modelo integra un equipo docente de profesores autores y tutores, ubicados en la sede central y centro de apoyo, los cuales gestionan el proceso educativo, con un conjunto de especialistas en la elaboración de materiales, evaluadores, expertos en contenidos, técnicos de informática y diseño gráfico (UTPL, 2018).

Este modelo se ajusta a los lineamientos curriculares que establece el Consejo de Educación Superior (CES); la malla curricular comprende 8 períodos académicos, con una cátedra integradora en cada período que articula varios de los contenidos estudiados en el ciclo. En este proceso los docentes orientan, acompañan y motivan de forma continua al autoaprendizaje en los estudiantes utilizando los recursos del Entorno Virtual de Aprendizaje y los demás herramientas tecnológicas (Sandoval, 2020).

Por lo tanto, los docentes deben buscar nuevas estrategias de apoyo pedagógico y didáctico como la clase inversa, el uso tecnología web 4.0, el aprendizaje basado en problemas y el aprendizaje basado en retos, para garantizar el dominio de las competencias y el aprendizaje significativo dada la diversidad de población estudiantil (Pamplona, Cuesta y Cano, 2019).

La integración es un proceso dinámico que permite la unificación de criterios, en función de satisfacer las necesidades académicas, sociales y empresariales mediante la incorporación de los estudiantes de pregrado a situaciones reales. Este proceso de integración debe tomar en cuenta satisfacer las demandas de los actores involucrados lo que requiere hacer adaptaciones a contenidos, enfoques, estructuras y estrategias basándose en la realidad actual (Hernández, et al. 2020).

La integración del currículo, es un proceso que se efectúa con la participación de un determinado número de disciplinas destinadas a cubrir un período temporal. Con esto no se trata sólo de enseñar un determinado contenido, sino además de motivar y desarrollar un conjunto de destrezas que facilitan nuevas relaciones entre estos y otros contenidos, en consecuencia, se genera una manera diferente de establecer vínculos con el conocimiento (Calneggia, et al. 2015).

En la actualidad, los centros educativos contemplan la integración como la incorporación de nuevos recursos didácticos. Puedes ser una asociación que se limita reforzar los modelos tradicionales transmisivos, unidireccionales de enseñanza 0 , en los centros que destacan por sus "buenas prácticas", una incorporación que conduce innovación educativa (González y Gutiérrez, 2017). 
Tomando en cuenta los aportes citados la integración curricular se realizó como estrategia para el desarrollo de competencias, habilidades y destrezas de los estudiantes de educación básica cursantes de las asignaturas Cátedra integradora 2: Diagnósticos de los contextos familiares y comunitarios, Neurociencia y psicología del desarrollo de la niñez y adolescencia y Planteamiento de la investigación acción participativa: Historias de vida y proyectos de vida. Para esto se elaboraron planes docentes sincronizados y secuenciados en función de los resultados de aprendizaje de cada asignatura y la solución del reto. Los estudiantes ejecutaron actividades en cada asignatura, así mismo, tenían claridad de la ejecución de un proceso sistémico porque las actividades se articulaban entre sí, no existía repetición de acciones, ni de temas, pues el objetivo final era la aplicación de las competencias académicas en la realidad.

Un elemento esencial que delimita el escenario de acción es el video de presentación, donde los tres docentes explican la integración de las tres asignaturas como un equipo con objetivos comunes. Los temas que articulaban el proceso fueron los contextos familiares y comunitarios, y el aprendizaje de los niños y adolescentes, de tal forma que los estudiantes pudieran comprender que la función docente implica diversas variables que debe conocer, analizar, interpretar en función de proponer acciones creativas para transformar aquellas limitaciones que impiden el aprendizaje significativo de los niños y adolescentes.

\section{Consideraciones metodológicas de la investigación}

La investigación responde al nivel del objetivo planteado, precisándose un estudio descriptivo el cual, según Rojas (2016:7) "exhibe el conocimiento de la realidad tal como se presenta en una situación de espacio y de tiempo dado. Aquí se observa y se registra, o se pregunta y se registra. Describe el fenómeno sin introducir modificaciones: tal cual".

Se abordaron los mecanismos idóneos que permitieron la integración de los elementos que conforman la estructura curricular, permitiendo de esta manera que la concepción educativa declarada en el currículo basado en el enfoque por competencias. En atención con esta premisa, el estudio se orientó bajo el enfoque cualitativo, que representa el procedimiento sistemático de indagación que ofrece técnicas especializadas para recabar datos.

La investigación es de corte interpretativa; se lleva a cabo en determinados grupos sociales, cuya participación es activa durante todo el desarrollo del proceso investigativo, a fin de conocer y generalizar la realidad natural de la comunidad (Escudero y Cortez, 2018).

La modalidad de investigación fue de campo de acuerdo con Escudero y Cortez (2018), tanto en el levantamiento de información, como el análisis, aplicaciones prácticas y métodos empleados para la obtención de resultados, se llevan a cabo en el medio donde se desenvuelve el evento investigado. Mediante la investigación de campo es posible efectuar manipulaciones controladas 
de una variable externa no verificada, con el propósito de describir las formas, así como las causas que originan determinada situación particular.

Desde esa perspectiva, se contó con la participación de tres docentes, 85 familias ecuatorianas, 70 tutores externos ubicados en diferentes ciudades de Ecuador y 85 estudiantes de segundo ciclo de la carrera de Educación Básica del departamento de Ciencias de las asignaturas Cátedra integradora 2: Diagnósticos de los contextos familiares y comunitarios, Neurociencia y psicología del desarrollo de la niñez y adolescencia y Planteamiento de la investigación acción participativa: Historias de vida y proyectos de vida.

Los estudiantes recorrieron trayectorias de aprendizaje integradas desde una etapa diagnóstica, a través el acercamiento a la comunidad, a los padres de familia y la aplicación de la técnica historia de vida; para luego determinar la problemática más relevante en relación con el aprendizaje de los niños y adolescentes.

Se aplicó el método de Investigación Acción Participativa (IAP), permitiendo a los participantes la experiencia de "aprendan a aprender", no solo en la problemática o necesidad encontrada, sino que desarrollan un punto de vista más crítico y desarrollan de habilidades que pueden aplicar posteriormente ante cualquier situación (Ortega, Ortega y Hernández, 2020).

El método IAP, permitió al profesorado la indagación de debilidades en la formación de la práctica docente, para promover el desarrollo de competencias en los 85 estudiantes de la carrera de Educación Básica, con el fin de potenciar su futuro desempeño docente o profesional.

En este sentido, el futuro profesional de la docencia debe integrar en su práctica la función investigadora como medio de autodesarrollo profesional e instrumento para mejorar la calidad de la enseñanza; a partir de esta premisa, la fase diagnóstica de esta investigación la ejecutaron los estudiantes; aplicando la técnica auto diagnóstica DAFO análisis de (debilidades, amenazas, fortalezas y oportunidades), dirigido a las familias objeto de estudio. Luego del análisis, intentaron dar respuesta a los siguientes nudos problemáticos, detectados:

- Falta de responsabilidad de los padres, en el proceso educativo de los hijos.

- Violencia en la convivencia familiar y escolar

- $\quad$ Carencia de vínculos afectivos entre los miembros de la familia, docentes y comunidad

- Bullyng escolar

- Escaso tiempo de dedicación a los hijos por parte de los padres

- Divorcio de la familia y en consecuencia desestructuración y dinámica familiar.

Los métodos de enseñanza, recursos empleados; así como la población objeto de estudio/ Actores involucrados se describen en el cuadro 1: 


\section{Cuadro 1}

Métodos, Recursos y Población

\begin{tabular}{cl}
\hline & Métodos, Recursos y Población \\
\hline & 1. Aprendizaje colaborativo \\
2. Uso de las tics & 3. Talleres \\
4. Clases explicativas sobre la secuencia didáctica para el & planteamiento de la estrategia de aprendizaje \\
\hline & 1. Software Pixton \\
& 2. Padlet para el muro digital \\
& 3. Zoom para las video colaboraciones \\
& 4. Matriz de autodiagnóstico DAFO \\
Recursos & educativas. \\
\hline Población objeto de estudio/: & 85 estudiantes de la carrera de Educación Básica. \\
\hline & 3 docentes de catedra \\
Actores involucrados & 70 tutores externos \\
& 85 familias ecuatorianas- \\
\hline
\end{tabular}

Fuente: elaboración propia (2019)

En relación con las actividades, estas se describen considerando recursos, responsables, tiempo, fecha, lugar de ejecución. En el cuadro 2, se plantean actividades de acuerdo a las 16 semanas que tiene el período académico, 8 del primer bimestre y 8 del segundo bimestre.

\section{Cuadro 2 \\ Actividades desarrolladas}

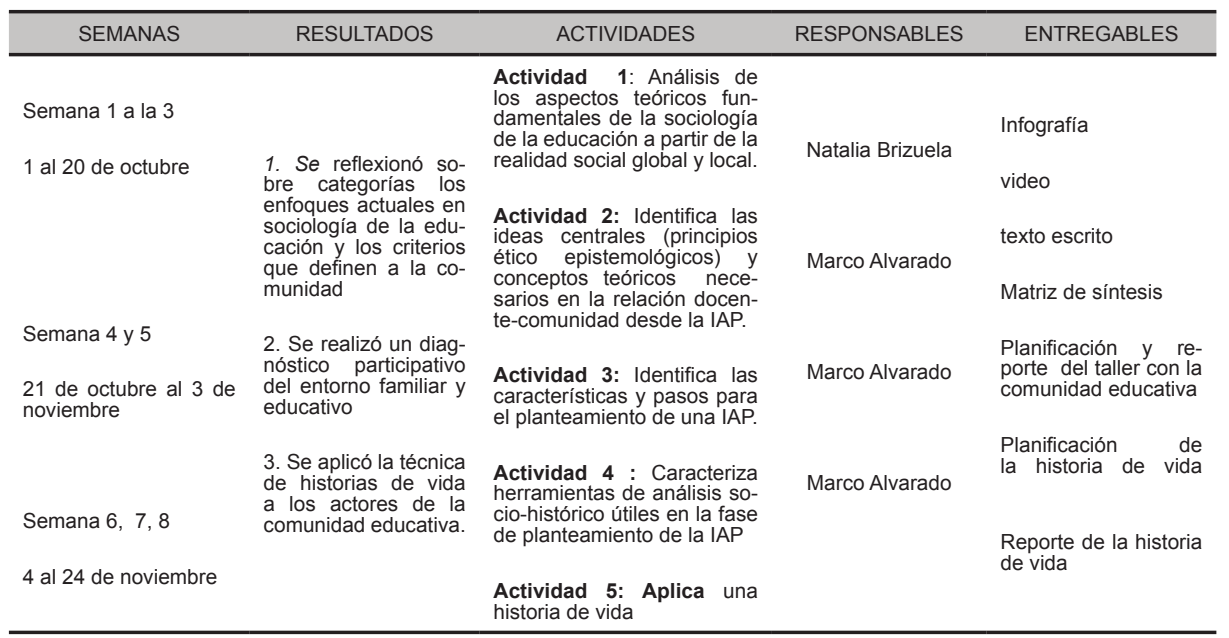




\section{Cont... Cuadro 2}

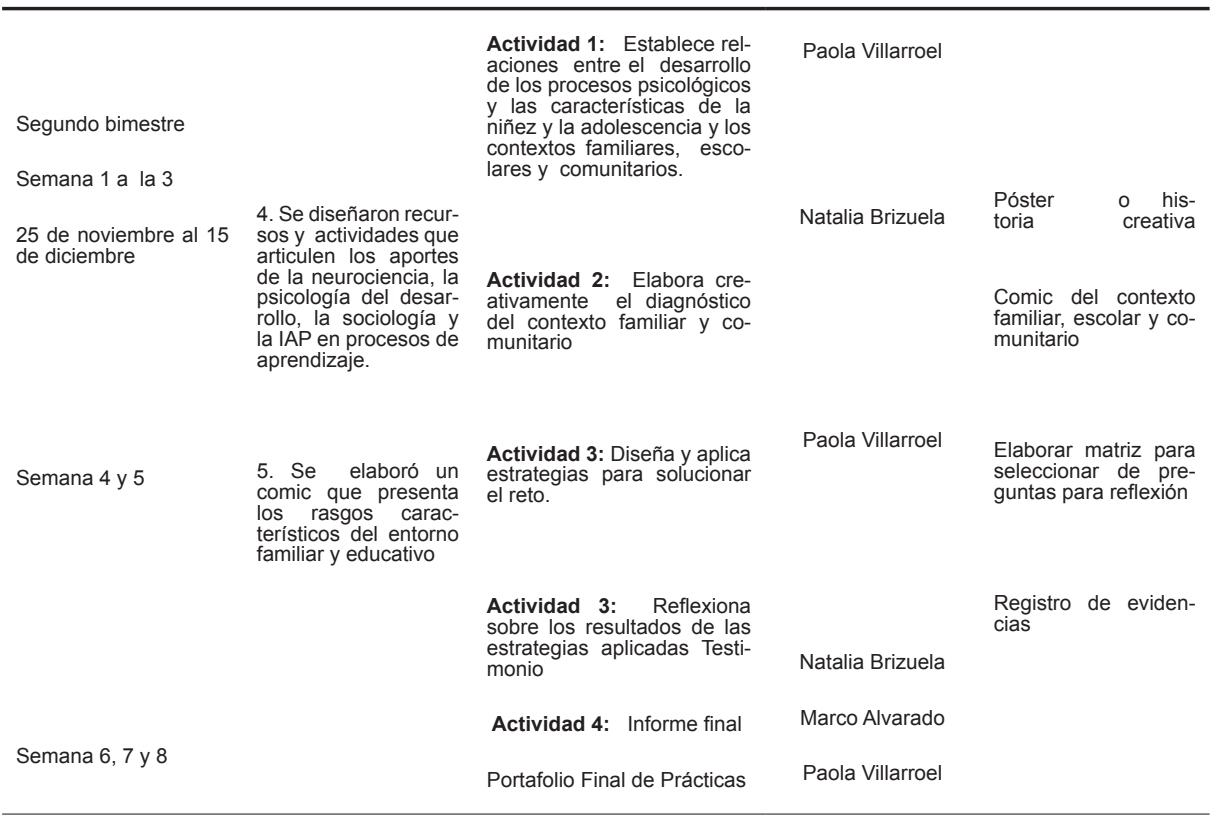

Fuente: elaboración propia (2019)

En función de la operatividad de la estrategia definida, se proyectó la interrelación positiva de los estudiantes de la carrera educación básica con los actores del contexto educativo (familia, escuela y comunidad). Para abordar esta estrategia, se articulan las asignaturas antes mencionadas, desde un enfoque sistémico que busca explicar la realidad de forma integral. Los estudiantes participantes deben aplicar la técnica de la historia de vida para elaborar un diagnóstico del contexto, a partir de la cual formulen propuestas de estrategias que aporten a los procesos de aprendizaje en los niños y adolescentes.

De ahí que se plantea la integración curricular como una forma de implementar el currículo que se fundamenta en la colaboración e interconexión de varias disciplinas para abordar diferentes situaciones o problemas de la realidad; promueve el desarrollo cognitivo de los estudiantes, mediante la identificación de problemáticas del contexto donde se desenvuelven, para encontrar soluciones oportunas e innovadoras (Molina, 2011).

En ese sentido, la comprensión del problema y la búsqueda de soluciones, implica aun análisis integral de la realidad, por lo cual no es adecuado desde la perspectiva curricular hacer este tipo de dinámicas solo desde una asignatura, sino integrar actividades y contenidos de varias asignaturas.

La propuesta se desarrolló en cuatro etapas, en el cuadro 3 se describen: 


\section{Cuadro 3 \\ Etapas de la estrategia}

Se realizaron las siguientes actividades de orden teórico como:

1. Analizar los aspectos teóricos fundamentales de la sociología de la educación a partir de la realidad social global y local, recurriendo a diferentes fuentes bibliográficas, análisis que fue abordado a través la Cátedra Integradora.

Acercamiento 2. La asignatura de planteamiento de la investigación acción participativa: Historias de vida y al contexto proyectos de vida facilitó la identificación de ideas centrales (principios ético epistemológicos) y conceptos teóricos necesarios en la relación docente-comunidad desde la Investigación Acción Participativa (IAP), así como las características, pasos y herramientas para el planteamiento de una IAP.

3. Planificación de un taller con los padres de familia, en el cual se aplicó la técnica de autodiagnóstico DAFO (análisis de debilidades, amenazas, fortalezas y oportunidades)

1. Los insumos obtenidos en el DAFO a través de la asignatura de Planteamiento de la investigación acción participativa: Historias de vida y proyectos de vida. Se complementan con la aplicación de la técnica de historias de vida que se aplicó en la Cátedra integradora para establecer las principales características de los contextos familiares y comunitarios Diagnóstico de desde la experiencia individual de los miembros de la comunidad educativa (padre o madre, problemática docente, niño/adolescente, miembro de la comunidad y estudiante).

2. Esta información se sistematiza desde un enfoque cualitativo, considerando las siguientes categorías: actores y relaciones de la comunidad educativa, comunicación, acompañamiento del aprendizaje, entre otros, cuyo resultado se presenta de forma creativa a través de la elaboración de un comic

Diseño de

estrategias

para el

aprendizaje

En esta fase, a partir del acercamiento y diagnóstico del contexto familiar se recurrió a los aportes de las neurociencias y psicología del desarrollo para diseñar y aplicar estrategias que pueden mejorar los procesos de aprendizaje de los niños y adolescentes con el apoyo de la familia, la escuela y comunidad.

1. Reflexión del trabajo realizado a través del testimonio de los estudiantes y los actores del proceso educativo: niños, adolescentes y padres de familia que evidenciaron el sentido de la experiencia para cada estudiante y el aporte para su propia vida y su entorno familiar y educativo.

2. Este testimonio y reflexión se presentó a través de un muro digital (padlet), creado con

Reflexión y testimonio de la experiencia los temas y actividades realizadas en las tres asignaturas y un video colaboración donde se presentaban las conclusiones respectivas

3. La evaluación del aprendizaje logrado en los estudiantes se realizó durante el período académico, a través de las actividades realizadas la matriz DAFO, el comic, la estrategia de aprendizaje con la participación de la familia, donde se aplicaron rúbricas específicas para su evaluación

4. Al final del ciclo se evalúo a través de la reflexión-testimonios de los estudiantes y los exámenes bimestrales.

\section{Integración Curricular y estrategias de aprendizaje: Resultados de la Carrera de Educación Básica, Universidad Técnica Particular de Loja en Ecuador.}

el aprendizaje de los estudiantes; a través, de la interrelación con la familia/ escuela / comunidad, a partir de la cual se proponen métodos para determinar las problemáticas más relevantes de los niños y adolescentes en sus contextos educativo o familiar.

En otro orden de ideas, a partir de 
Varguillas, Carmen; Brizuela Camacho, Sor Natalia; Villarroel Dávila, Paola Carina y Gallardo V., Daniel Armando

Estrategias de aprendizaje e integración Curricular en la Universidad Técnica Particular de Loja en Ecuador

la aplicación del DAFO por parte de los estudiantes participantes y la historia de vida de las familias abordadas, se estableció el diagnóstico de los contextos familiares (cuadro 4) que se sintetizan en los siguientes aspectos:

\section{Cuadro 4}

\section{Debilidades, Amenazas, Fortalezas, Oportunidades (DAFO)}

\section{Aspectos encontrados por los estudiantes a partir del DAFO}

1. Divorcios y abandono del padre, frente a lo cual la madre debe asumir el rol de formación y sustento económico para la familia, lo que provoca sentimientos de desconfianza, inseguridad.

2. Dificultades de aprendizaje como dislalia funcional, hiperactividad y discapacidad que no fueron aceptadas en el contexto escolar, pero con el apoyo de la familia se lograron superar.

3. Migración a las grandes ciudades.

4. Maltrato y violencia intrafamiliar de padres a hijos.

5. En la etapa de la adolescencia se observó con mayor frecuencia situaciones de bullying, rebeldía y embarazo precoz.

Fuente: elaboración propia (2019)

Las problemáticas obtenidas en el cuadro 4 , fueron representadas (figura
1) a través de un comic:

\section{Comic del contexto escolar y comunitario}

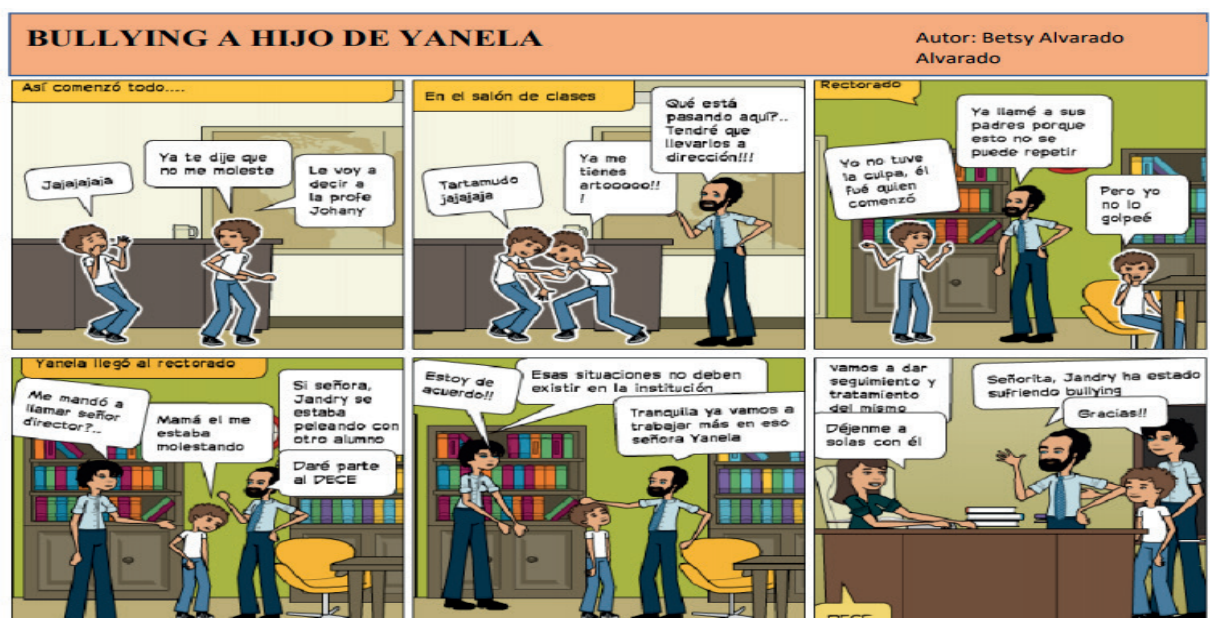

Fuente: T. Alvarado (Comunicación personal, 10 julio 2020) 
En el diseño de estrategias de aprendizaje, se priorizó la creatividad de los estudiantes para proponer actividades innovadoras que fueran pertinentes en la atención de la problemática detectada. El diseño de la estrategia incluyó los siguientes aspectos:

- Justificación de la realización de la estrategia:

- Objetivos:

- Habilidad/es o destreza/s desarrollada/s.

- Recursos:

- Descripción de la estrategia

- Análisis reflexión de la aplicación de la estrategia.

En la aplicación de la estrategia de acercamiento de los estudiantes hacia los contextos reales se observaron los resultados presentados en el siguiente cuadro 5 :

\section{Cuadro 5 Acercamiento estudiantes-contexto}

\begin{tabular}{l}
\hline Resultados del acercamiento de los estudiantes con el contexto real \\
\hline Los estudiantes comprenden los fundamentos teóricos de la neurociencia y la psicología del de- \\
sarrollo para diseñar estrategias que potencien las habilidades cognitivas, socioafectivas y motrices. \\
Los estudiantes reflexionan sobre el rol del docente en el proceso de aprendizaje de los niños y en \\
la comunicación con la familia gracias a la interacción directa con la realidad escolar o familiar. \\
- Los estudiantes identifican los aspectos positivos de la aplicación de las estrategias y aquellos \\
aspectos que se deben mejorar debido a las variables que condicionan el proceso educativo. \\
porque se requiere mayor acompañamiento a los estudiantes en las diferentes actividades.
\end{tabular}

Fuente: elaboración propia (2019)

\section{Conclusiones}

El aprendizaje basado en retos vincula la teoría y práctica a través de la aplicación de instrumentos de investigación acción participativa y estrategias de aprendizaje en los contextos escolares y familiares, por lo que los estudiantes identifican la importancia del conocimiento teórico para garantizar acciones efectivas en la realidad objetiva y transformarla.

Los estudiantes valoran, a través de sus testimonios, la importancia de la integración de las tres asignaturas para su aprendizaje porque supera los esquemas tradicionales y convierte al proceso de enseñanza aprendizaje en un sistema integral, que no fragmenta el conocimiento en islas de información, por el contrario intenta articular e incidir de manera significativa en la formación del estudiante y en el trabajo con niños, adolescentes y padres de familia.

Finalmente, el proyecto durante todas las etapas, provocó el acercamiento de los estudiantes hacia los contextos reales para intervenir de forma directa con los actores del proceso educativo, permitiendo desarrollar habilidades de observación, descripción, análisis y cuestionamiento. 
Varguillas, Carmen; Brizuela Camacho, Sor Natalia; Villarroel Dávila, Paola Carina y Gallardo V., Daniel Armando

Estrategias de aprendizaje e integración Curricular en la Universidad Técnica Particular de Loja en Ecuador

\section{Referencias bibliográficas}

Calneggia, M., Lucchese, M., Di Francesco, A. y Benítez, D. (2015). Integración Curricular y Procesos de investigación acción entre Universidad y Escuela Secundaria. Conference Paper. Universidad Católica de Córdoba -Facultad de Educación. https://bit.ly/3dqj/4E

Casanova, I., Paredes, I., y Ortega, E. (2020). Ejes transversales y perfiles por competencia: Una propuesta viable para su ejecución. TELOS: Revista de Estudios Interdisciplinarios en Ciencias Sociales, 22(3), 510-527. www.doi.org/10.36390/telos223.03

Escudero, C. y Cortez, L. (2018). Técnicas y métodos cualitativos para la investigación científica. 1ra. ed. Editorial UTMACH. $\quad$ http://186.3.32.121/ bitstream/48000/12501/1/Tecnicasy-MetodoscualitativosParalnvestigac ionCientifica.pdf

Fernández, M. (2004). El desarrollo docente en los escenarios del currículum y la organización. Revista de currículum y formación del profesorado, 8(1), 1-20. https://www. ugr.es/ recfpro/rev81COL3.pdf

González, R y Gutiérrez, A. (2017). Competencias mediática y digital del profesorado e integración curricular de la tecnologías digitales. Revista Fuentes, 19(2), 57-67. https:// revistascientificas.us.es/index.php/ fuentes/article/view/4028

Granados, E. (2014). El currículo de la educación superior desde la complejidad sistémica. Guayaquil, Guayas, Ecuador: CES.

Hernández, G; Mármol, M; Noriega, J; Ramos, E y Faría, G. (2020). Integración: educación universitaria, Estado y sector productivo mediante las prácticas profesionales. Revista
Negotium, 46(16), 19-29.

Illán, N., y Molina, J. (2011). Integración Curricular: respuesta al reto de educar en y desde la diversidad. Revista Educar em, 17-40. https://bit. ly/3x4p7pS

Malmqvist, J., Rådberg, K; Lundqvist, K. (2015). Comparative Analysis of Challenge-Based Learning Experiences. Proceedings of the 11th International CDIO Conference, Chengdu University of Information Technology, 1-13.

Observatorio de Innovación Educativa del Tecnológico de Monterrey. (2015). Aprendizaje basado en retos. Eduteka. http://Eduteka.Icesi.Edu. Co/Articulos/Edutrends-10-2015

Ortega, I., Ortega, E., y Hernández, A. (2020). La investigación de acción participativa: ¿una alternativa deinvestigación o una estrategia de solución? Centro de Investigación en Alimentación y Nutrición (CINA). Universidad del Istmo (UNISTMO), campus Juchitán de Zaragoza. Oaxaca. México. https://bit. $\underline{\mathrm{ly} / 3 \mathrm{qxG} 2 \mathrm{PO}}$

Pamplona, J., Cuesta, J., y Cano, V. (2019). Estrategias de enseñanza del docente en las áreas básicas: una mirada al aprendizaje escolar. Revista Eleuthera, 21, 13-33. https:// doi.org/10.17151/eleu.2019.21.2

Rojas, M. (2016). Tipos de Investigación científica: Una simplificación de la complicada incoherente nomenclatura y clasificación. REDVET. Revista Electrónica de Veterinaria, 16(1), 1-14. https://www. redalyc.org/pdf/636/63638739004. pdf

Sandoval, C. (2020). La Educación en Tiempo del Covid-19 Herramientas TIC: El Nuevo Rol Docente en el Fortalecimiento del Proceso 
pp. 818-831

Revista Venezolana de Gerencia, Año 26 No. 95, 2021

Enseñanza Aprendizaje de las Prácticas Educativa Innovadoras. Revista Tecnológica-Educativa Docentes 2.0, 9(2), 24-31. https://doi. org/10.37843/rted.v9i2.138

Universidad Técnica Particular de Loja. (2018). Modelo Educativo Institucional de la UTPL. https:// procuraduria.utpl.edu.ec/sitios/ documentos/NormativasPublicas/ Resoluci\%C3\%B3n\%20Modelo\%20 Educativo.pdf
Zamudio, A., Leiva, S., y Fernández, M. (2019). Integración Curricular: Un Proceso De Investigación Acerca Del Curriculum Universitario. Revista De La Escuela De Ciencias De La Educación, 2(14), 28-40. https:// revistacseducacion.unr.edu.ar/index. php/educacion/article/view/447 